\title{
Brain Computer Interface for controlling mobile Robot
}

\author{
Hareendar S, Jeya raghul R, Aashish kumaravelan
}

\begin{abstract}
This Paper describes a brainwave controlled mobile robot based on brain computer interface (bci). This uses Matlab to analyze the brainwaves. Bci bypass the communication path between brain and computer to establish a communication system in which the data flows directly from brain to physical devices. Bci consists of software and hardware components that allow brain inputs to be translated as controls for machine. The different brain patterns are translated into different machine commands. These obtained commands are used to control the mobile robot. This technology is widely used in neuro-prosthetic devices, which helps people in achieving or improving their capability. . Our intention in this paper is to develop a smart wheelchair which will help disabled people in their mobility. The Bci consists of Neurosky brainwave headset which is connected to computer for extracting the data and to process real time commands, which is then used to control the module.
\end{abstract}

Index Terms: Brain computer interface (Bci), Neurosky, Matlab

\section{INTRODUCTION}

Robots are widely used for industrial applications, but are now gradually used in rehabilitation of patients. There is a growing demand for the assisting robots as it provides aid for disabled person in their daily life. Generally healthy people are capable of operating robots using traditional input devices like mouse, keyboard or a joystick but people with severe illness like amyotrophic lateral sclerosis (ALS), multiple sclerosis (MS) or strokes cannot operate those input device, Even an autonomous robot doesn't provide precise output. Thus To bring a complete solution for the problem, bci was developed [1, 3]. They are made to analyze human brain activities with respective brain wave signals. With respect to different frequencies, brainwaves are classified into gamma, beta, alpha, theta and delta [2]. In our paper, we design a robot module which is controlled by an EEG based bci.

The bci performs extraction, processing and classification of raw EEG signals. After the preprocessing and analysis of the signals the threshold values are obtained. The respective commands are generated accordingly with the threshold values which drive the motor. Thus design of controlling mobile robot can lead to a construction of smart wheelchair which assists disabled people in mobility.

Revised Manuscript Received on July 05, 2019.

Hareendar S, ECE department, SRM institute of science and technology, kancheepuram,India.

Jeya raghul R, ECE department, SRM institute of science and technology, kancheepuram,India.

Aashish kumaravelan ECE department, SRM institute of science and technology, kancheepuram,India.

\section{LITERATURE REVIEW}

To provide assistance to disabled people brain computer interface (bci) is developed. Electroencephalogram (EEG) is a conduction of measurement process to detect the electrical activity of the brain which is caused by neural synaptic excitations, EEG signals are basically recorded with scalp electrodes, a very poor quality of signals are obtained due to noise which are unavoidable element produced either inside or outside on the scalp and skull thickness, thus the signals are amplified [1].The EEG can be classified into different frequency ranges based on the power spectrum of the brain signals. Delta, theta, alpha, beta, gamma are the different frequency ranges [2].The brain -computer interface is mainly developed to provide the adequate commands for the operation, controlling machines with thought power, the brainwaves are picked up, detected and translated into sequence of commands, restoring ability of the patients is considered the main task of the BCIs to make the patients live their day to day life. Each bci consists of four important elements; recording the raw EEG data, extraction of important information from raw signal, comparing the signal with neural activity patterns to identify the brain activity, generating commands according to brain activity the user performs [4]. The mobile robots can be directly controlled by several kind of brain signals like steady state visual evoked potential (SSVEP), event related desynchronization/event related synchronization (ERD/ERS), P300. BCI system which has single signal may not work for all people. Certain users cannot provide needed brain activity pattern for the BCI. For e.g.: people responding to brain signals for ERD BCI could not produce required brain signal patterns for an SSVEP based BCI.[3]

\section{EXISTING SYSTEM}

In general, mobile robots are controlled by conventional input devices like mouse, keyboard or joystick. The input to the conventional devices is guided by an interface, however elderly and disabled people have difficulty with operating these input devices. Thus, special interface like sip-and-puff system, eye tracking, single type switches autonomous robot were used. The sip-and -puff technology works on air pressure by inhaling and exhaling. It basically consists of mouthpiece with straw to blow, which is connected to dual pressure switch. In sip-and puff the movement of limbs is not required. It has certain drawbacks like maintenance of the straw/tubing proper lip closure of the patient is required; user needs to have the ability to follow multi step commands.

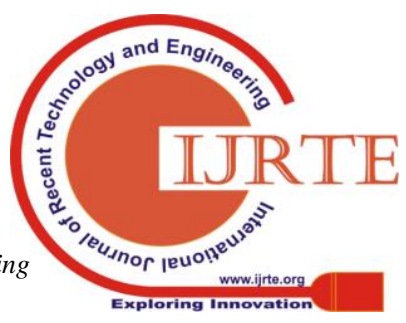


In eye tracking, the system uses eyeball movement to move wheelchair. The detection of eyeball is done by capturing it through web-cam fixed in the user spectacles. The image captured is sent to the micro controller for digital image processing, and the output is based on the motion direction of the eyeball. These are not much used due to its high cost, and people with eye defects such as myopia and hypermetropia as there are forced to wear corrective spectacles, and also calibration of equipment takes time. In Autonomous wheelchair there is a mismatch in output with the intention of the patient. Currently EEG acquisition system are widely used for controlling the robot, which traditionally had invasive electrodes implanted inside the skull, later non-invasive multi dry scalp electrodes are used to detect the brainwaves.

\section{PROPOSED SYSTEM}

This proposed system consists mainly of the brainwave sensor, a personal computer, ZigBee module, Arduino Uno and a motor driver (L293D). Initially, the patient is made to wear the Neurosky headset. The parts of the brainwave sensor are shown in Fig.1.[5,7]. Our proposed system is implemented with a non-invasive BCI system, where the dry electrode is positioned on the forehead of the user, exactly at the FP1 location (10-20 electrode system) as shown in the Fig.2 [6]. Unlike the other EEG headsets having more than one electrode location. This sensor provides reduced complexity and increased accuracy, which contains think gear chip within it for pre-processing. This dry electrode acquires the EEG signals and are amplified and filtered to remove power line noise and frequency related body movement noise.

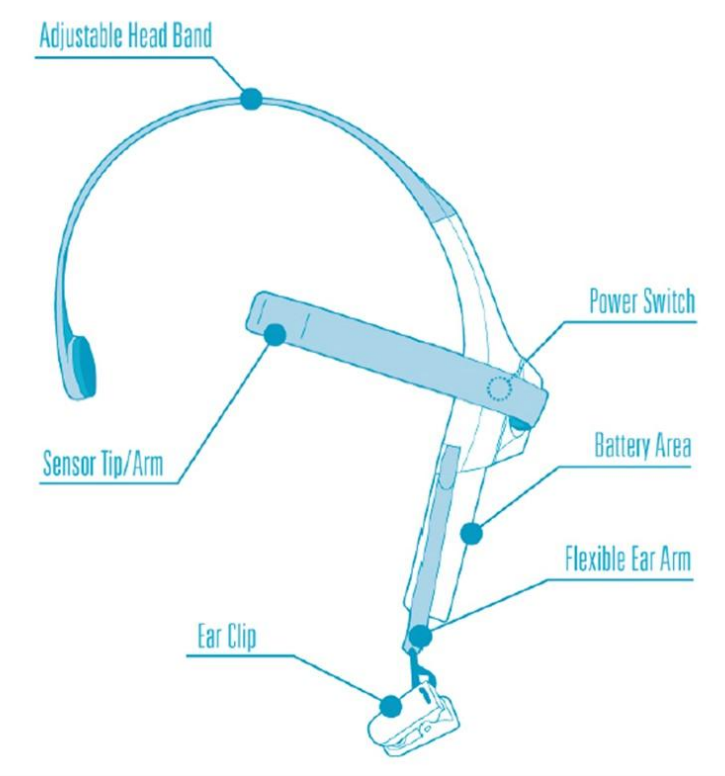

Figure 1: Brainwave sensor

This raw EEG signal is sent to the personal computer and the required feature is extracted using a Matlab program. The attention level, meditation level, and the blink strength are calculated periodically and also plotted in a graph for easy observation. The data is then transmitted and received via the
ZigBee module which is then connected to the Arduino. The Arduino UNO is then embedded with a program to drive the robot module with the help of the motor driver.

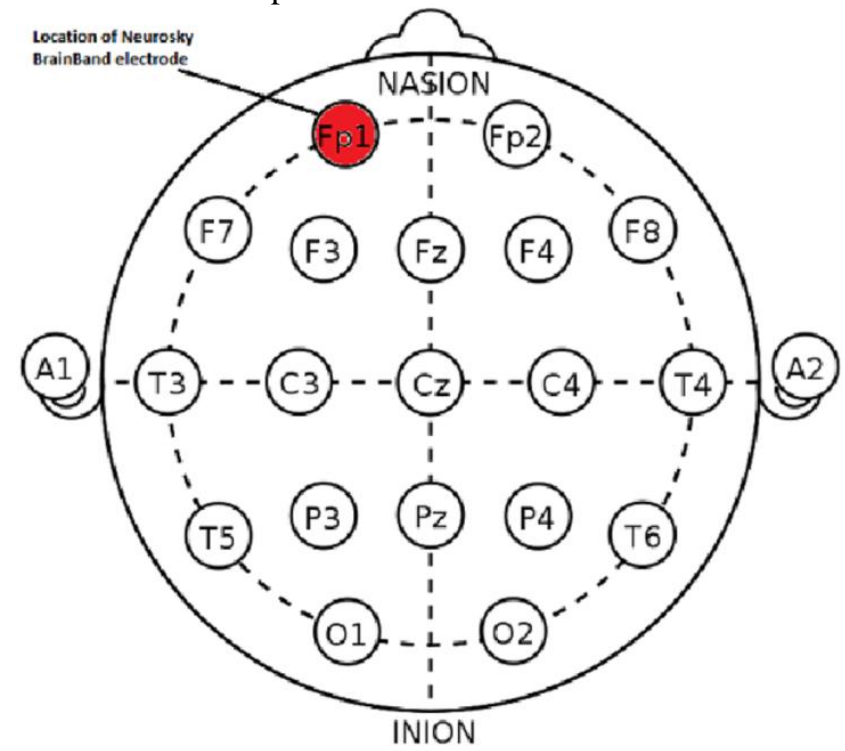

Figure 2: Electrode position

\section{DESCRIPTION}

The block diagram shown below describes the entire brain computer interface system. The brainwaves generated due to the neuron activity are captured by the single dry electrode. The obtained brainwave signals are initially pre-processed to remove problematic elements like body movement noise. Then the preprocessed signals are amplified and this signal is transmitted as a raw data to the Data processing unit via Bluetooth module. In the data processing unit, the computer receives the raw data from the brainwave sensor via Bluetooth module.

The raw data is then extracted and the required information such as the attention level, meditation level and the blink strength are obtained with a Matlab program periodically and are also plotted in a graph for easy observation.The threshold values are fixed for each factor and the signal is constantly monitored for the threshold values .when the fixed threshold values are obtained; they are transmitted to the ZigBee as a serial data via USB-TTL cable and then transmitted to the Arduino board. The Arduino is programmed to receive the threshold values and generate sequence of commands accordingly. The command is then passed on to the motor driver which drives the robot. 

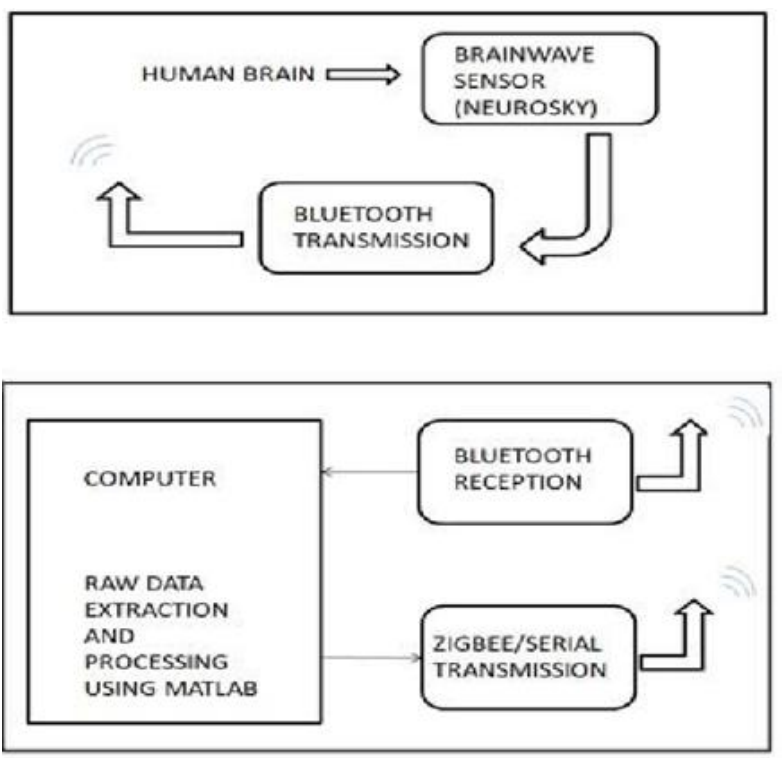

Figure 3: Brain computer interface

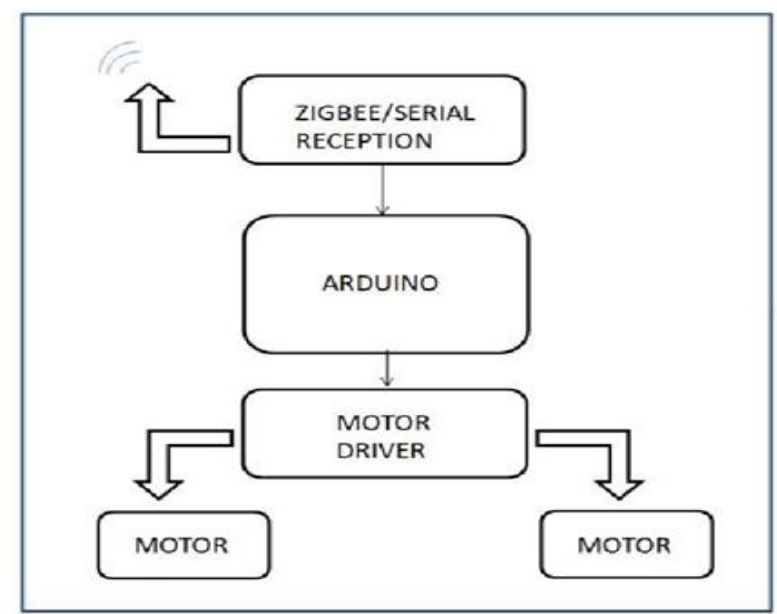

Figure 4: Robot module

\section{A. FLOW CHART}

Initially the brainwaves of different frequencies namely delta, theta, alpha, beta, gamma shown in the Figure 5 are obtained by the Neurosky sensor. The ranging values are then classified as meditation level, Attention level inside the sensor with the use of built-in chip.[12]

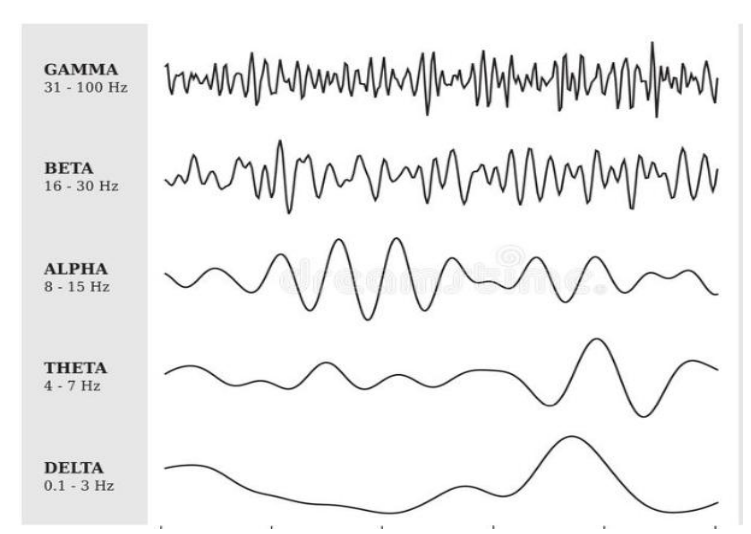

Figure 5: brainwave frequencies
The Neurosky is then connected to the personal computer to transfer the data in Matlab for further process. A specific Matlab code is used for processing the signal to obtain respective control signals. The control signals or the commands are transferred to the Arduino via ZigBee transceiver. The Arduino then with the help of motor driver drives the robot based on the given commands. The methodology is shown in Fig 6.

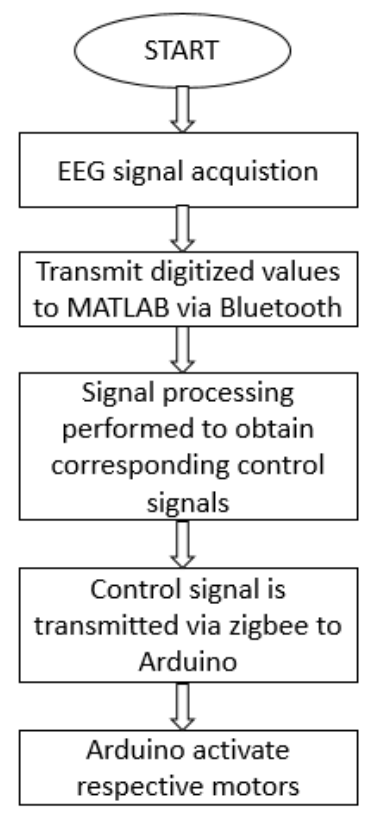

Figure 6: Methodology

The flowchart shown in Fig. 7 describes the algorithm of the project. The eye blink is enabled and when it is triggered, it passes on to next block for checking attention and meditation level and the required threshold values are fixed for each movement. Then after the termination signal or the manual termination the process is stopped.

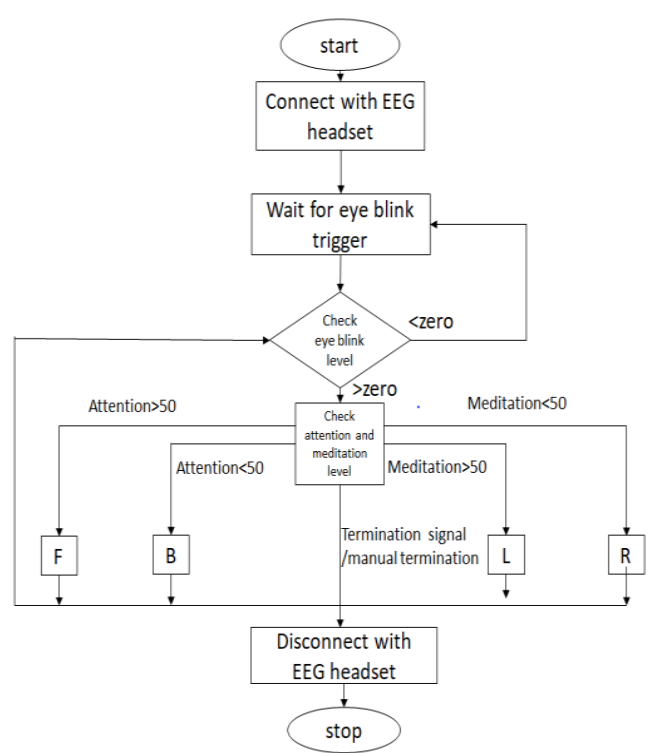

Figure 7: Flowchart 


\section{B. HARDWARE DESCRIPTION}

\subsection{Brainwave Sensor}

NEUROSKY EEG biosensor is non-invasive type, which has a single dry electrode placed on the selected FP1 position of 10-20 electrode system. This device can predict the brainwave and measures the attention, meditation level and blink level strength. All Neurosky sensor products enclose a Think Gear chip that enables that works as a link between human brain and the robotic system. Its value ranges from 0-256 and operating frequency $0.050 .5 \mathrm{~Hz}$ [12]. Some Specifications of Neurosky include its frequency range from 2.42- $2.472 \mathrm{GHz}$ and its power is $50 \mathrm{~mW}$ with a Sampling rate of $512 \mathrm{~Hz}$ for the EEG signal.

\subsection{Arduino}

Arduino is basically an open-source platform most commonly serving the purpose of building electronics projects. It consists of a board with inbuilt microcontroller programmed using software or IDE (Integrated Development Environment). Using an Arduino allows the connection of a USB to it rather than using a separate code to run external information or hardware. Also the programming used in Arduino is usually embedded $\mathrm{C}$ which makes it user friendly and much simpler to under-stand and use. Different kinds of components can be used and attached to the Arduino board like the various sensors along with GPS (global positioning system), GSM modules (global system for mobile communication) etc. according to the requirements.

\subsection{ZigBee}

ZigBee is used to create personal area networks for the connection with the $\mathrm{pc}$ and the robot vehicle. It is preferred as it has low-power digital radios. It consumes low-power and operates at low bandwidth and close proximity (10 to 20 meters). The ZigBee is less expensive compared to other wireless personal area networks. They operate in the frequency range of $2.4 \mathrm{GHz}$ for all industrial, medical or scientific purposes. The networks use 128 bit symmetric key encryption to make the data secured .The ZigBee is used to transfer the data from the personal computer to the Arduino.

\subsection{Motor driver}

Motor drivers are basically used to drive the motors, they take input signal with low current and amplify it to give high current signal .The motor driver L293D has two H-bridge driver circuits built in. For the operation of motors pins 9 and 1 are used, when the pins are set high driver becomes enabled, making the output to on. When the pins are set low the driver disables turning it off. The power supply of $12 \mathrm{v}$ is provided directly via the lithium polymer battery, the motor driver is then directly connected to the Arduino, and the control signals are obtained.

\subsection{Dc motor}

The back of the robot module consists of two $12 \mathrm{v}$ dc motors, each with 100 RPM and their wheels are coupled with the dc motors. For the power supply one $12 \mathrm{v} 2400 \mathrm{mAh}$ lithium polymer battery is used.

\section{SOFTWARE DESCRIPTIONS}

\subsection{Matlab}

Matlab is software used to obtain the threshold values from the brainwaves with the developed code. Matlab is also extensively used for other math and computation also for simulating and visualization.

\subsection{Arduino IDE}

An Arduino IDE is open source software that is used to write programs which are embedded to the Arduino board to obtain widespread outputs majorly used for student projects. It works on Windows, Mac and Linux. It is very easy to install and use.

\section{RESULTS}

First, in the Matlab program buffer is pre allocated for the blink strength level, attention level and meditation level, and the respective port number is defined for the Bluetooth receiver connecting the brainwave sensor. The think gear library is loaded to obtain raw data from brain wave sensor. This program reads the packet from the raw data for each factor, for every cycle and the loop continues until we obtain threshold values. If the blink level is greater than zero the system is triggered.

Then if the attention level is greater than 50 it displays $\mathrm{F}$ and if less than 50 displays B similarly if the meditation level is greater than 50 it displays L and meditation level less than 50 it displays $\mathrm{R}$. These values can be altered as required for the user by trial and error.

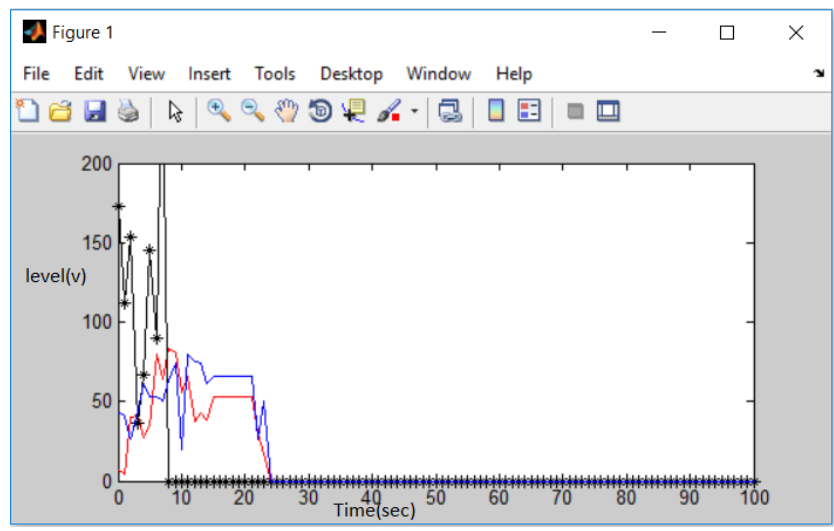

Figure 8: Output Graph

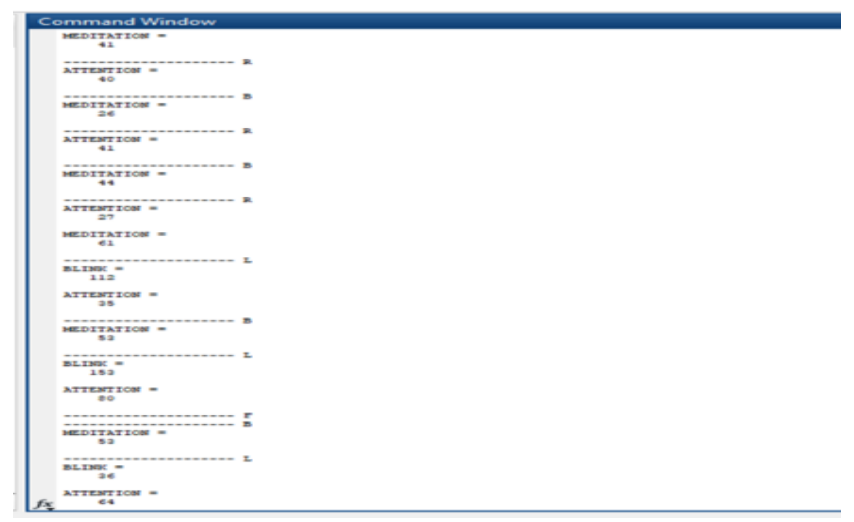

Figure 9: Matlab output 
The obtained values for each level are plotted in graph shown in Fig.8. The obtained commands in Matlab are shown in Fig.9. The wave forms depend on the user level of the thinking that the brainwave sensor captures.

Second, the Arduino is programmed depending on the threshold value data as F, B, L and R correspondingly as Forward, Backward, Left and Right which the motor driver works based on the command from the Arduino program, for moving forward, backward, left and right.

\section{CONCLUSION}

This project mainly focuses to build a very established and functional BCI controlled mobile robot mechanism which works using Neurosky headset. In the Matlab program the threshold values were successfully obtained and the commands given to the Arduino are executed successfully to drive the motor, hence controlling of mobile robot using BCI is successfully achieved. This technique is helpful in assisting the disabled people. This project leads to a development of smart wheelchair in future. BCI is highly progressing multidisciplinary field; its growth is fasten by research advancements in computer science, neuroscience, neurology, rehabilitation. Thus BCI hold promises in future rehabilitation engineering such as treating all kind of disabilities ,emotional disorders and even establishing two way communication ex operating robotic arm using brain signals from brain with sensation sent back into brain as signals .

\section{REFERENCES}

[1] Ms Priyanka D. Girase, Prof. M. P. Deshmukh “A Review of Brain Computer Interface" International Conference on Global Trends in Engineering, Technology and Management (ICGTETM-2016).

[2] Busra ulker, Mehmet Baris Tabakcioglu, Huseyin cizmeci, Doruk Ayberkin "Relations of attention and meditation level with learning in engineering education" ECAI 2017 - International Conference 9th Edition ,IEEE,1-4. https://doi.org/10.1109/ECAI.2017.8166407

[3] Luzheng Bi, Member, IEEE, Xin-An Fan, and Yili Liu, Member, IEEE "EEG-Based Brain-Controlled Mobile Robots: A Survey" IEEE TRANSACTIONS ON HUMAN-MACHINE SYSTEMS, VOL. 43, NO.2 MARCH 2013. https://doi.org/10.1109/TSMCC.2012.2219046

[4] Ewelina Sobotnicka, Aleksander Sobotnicki "BCI interface new opportunities and hopes for the disabled. An overview of available solutions" 2018 Baltic URSI Symposium (URSI). https://doi.org/10.23919/URSI.2018.8406697

[5] MindWave Mobile : User Guide, NeuroSky Inc., August 2015.

[6] N. H. Liu, C. Y. Chiang, H. C. Chu, "Recognizing the degree of human attention using EEG signals from mobile sensors", Sensors, vol. 13, no.8, $\quad$ pp. 1027310285, 2013 https://dx.doi.org/10.3390\%2Fs130810273

[7] NeuroExperimenter Users Guide, NeuroSky Inc., September 2015.

[8] Abdulkader, S. N., Atia, A., and Mostafa, M.-S. M. (2015). "Brain computer interfacing:Applications and challenges." Egyptian Informatics Journal, 16(2), 213-230. https://doi.org/10.1016/j.eij.2015.06.002

[9] Bi, L., Fan, X.-A., and Liu, Y. (2013). "Eeg-based brain-controlled mobile robots: a survey." IEEE transactions on human-machine systems,
https://doi.org/10.1109/TSMCC.2012.2219046

[10] Dev, A., Rahman, M. A., and Mamun, N. (2018). "Design of an eeg-based brain controlledwheelchair for quadriplegic patients." 2018 3rd International Conference for Convergence in Technology (I2CT), IEEE, 1-5. https://doi.org/10.1109/I2CT.2018.8529751
[11] Kanaga, E. G. M., Kumaran, R. M., Hema, M., Manohari, R. G., and Thomas, T. A.(2017). "An experimental investigations on classifiers for brain computer interface (bci) based authentication." 2017 International Conference on Trends in Electronics and Informatics (ICEI), IEEE, 1-6. https://doi.org/10.1109/ICOEI.2017.8300873

[12] Lawpradit, W. and Yooyativong, T. (2018). "The eeg brain signal representation forsurfaces and shapes touching behavior with an inexpensive device." 2018 International ECTI Northern Section Conference on Electrical, Electronics, Computer and Telecommunications Engineering (ECTI-NCON), IEEE, 135-140. https://doi.org/10.1109/ECTI-NCON.2018.8378297

[13] Stephygraph, L. R., Arunkumar, N., and Venkatraman, V. (2015) "Wireless mobile robot control through human machine interface using brain signals.” 2015 International Conference on Smart Technologies and Management for Computing, Communication, Controls, Energy and Materials (ICSTM), IEEE, 596-603. https://doi.org/10.1109/ICSTM.2015.7225484

\section{AUTHORS PROFILE}

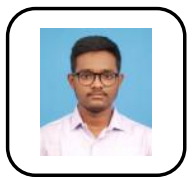

Hareendar S ,B.Tech in Electronics and communication engineering., area of interest include embedded systems, satellite communication

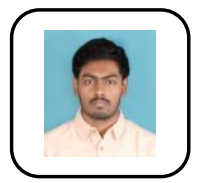

Jeya raghul $\mathbf{R}$,B.Tech in Electronics and communication engineering., area of interest include embedded systems and digital electronics.

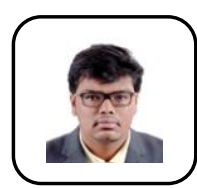

Aashish kumaravelan ,B.Tech in Electronics and communication engineering., area of interest include telecommunication ,network security and engineering management. 